\title{
MICROSTRUCTURING OF MULTIPHASE FOOD SYSTEMS IN SHEAR AND ELONGATIONAL PROCESS FLOWS
}

\author{
Prof. Dr. Erich J. Windhab, \\ Dr. Peter Fischer, Dr. Michael Stranzinger, Stefan Kaufmann \\ Swiss Federal Institute of Technology Zürich (ETH), Institute of Food Science; \\ Laboratory of Food Process Engineering; CH-8092 Zürich, Switzerland
}

\begin{abstract}
A large variety of food systems is treated in dispersing operations in order to generate a homogeneous disperse microstructure which allows to adjust structurerelated quality characteristics of the final products. For fine dispersing of disperse structures like solid particles/biological cells, emulsion drops, gas cells and aggregates thereof, the volumetric power and energy inputs are the crucial integral processing parameters but are not sufficient in order to optimize the dispersing efficiency. The flow field characteristics are of additional importance. For most concentrated food systems containing a disperse phase, the dispersing flow field in any apparatus is laminar. However the ratio of shear and elongational laminar flow contributions influences the dispersing efficiency strongly. Beside disperse components most multiphase food systems contain also macromolecular stabilizing components in the continuous phase as well as surfactants at the interfaces. The various disperse and macromolecular components have in general different sensitivity to mechanical stresses acting in dispersing flows.

Consequently, there is a need to optimize dispersing flow apparatus and adapt the stresses acting during processing in order to adjust the resulting microstructure. The most important structural characteristics for the disperse phase(s) are size, size distribution and shape which have a strong impact on the rheology of the fluid system and which are preferably focussed within the work reported here.

It is shown that in well defined shear and/or elongational dispersing flow fields the disperse particle size distribution and shape can be adjusted in order to generate specific rheological and quality properties of related food systems. It is also demonstrated how the synergistic application of dispersing flow experiments and Computational Fluid Dynamics (CFD) allow to optimize dispersing flow processes and related product properties.
\end{abstract}

\section{INTRODUCTION}

Micro-structuring in shear- and elongational dispersing flow fields links the areas of process engineering and material engineering. The latter includes the structure- rheology relationships. Rheologically the viscous behaviour of 
multiphase food systems is in general non-Newtonian, mostly shear-thinning and time-dependent. In many cases elastic properties are also not negligible. Dispersing as a flow structuring unit operation regarded here, generates the product microstructure due to shear- and normal stresses which act in the dispersing flow velocity field. A detailed description of the velocity field can be received from flow visualization experiments and from Computational Fluid Dynamics (CFD). The coupling of local velocity field information and rheological material functions which are received from rheometric shear and elongation experiments, allow for determining the related local shear and normal stress distributions. Microstructuring is triggered by the stresses acting locally in the flow field and occurs in general if critical stresses of the "structuring units" like networks, droplets, particle aggregates are exceeded. In order to generate a flow-induced structure the structuring units have to experience along their flow tracks a certain total deformation which requires a sufficient shear-/elongational rates and residence times.

\section{STRUCTURE RHEOLOGY RELATIONSHIPS}

Laminar flow fields are of uni- or multiaxial shear or elongational nature or a mixture of both. Within such flows, the rheological behaviour mirrors the dynamic behaviour of structural units which can change by orientation, deformation and aggregation or de-aggregation (= structuring mechanisms). The latter is synonymous to dispersing.

Figure 1 gives a schematic overview on such structure rheology relationships related to the viscous behaviour in shear flow. The flow induced structuring generates non-Newtonian behaviour even for dispersions with Newtonian continuous fluid phases. The shear rate-, disperse phase concentration- and time dependent viscous behaviour can be described by the structure-related fluid immobilization behaviour, which depends strongly on the structuring mechanisms described before and investigated in previous work [1].

Elongational flow fields induce more efficiently micro-structural changes due to the fact that there is no rotational contribution of the velocity field which, like in shear flows, causes structuring units to rotate. Rotation prevents structuring units from being strongly deformed, because the acting stresses may not act long enough to exceed a critical deformation. This is the more the case if the structuring units are highly viscous or of solid-like nature. This is demonstrated in figure 2 for the breakup of droplets with the diameter $\mathrm{x}$ in uniaxial shear- or elongation flows by the well known critical Weber number We- ( see equation 1) dependency from the viscosity ratio $\lambda$ ( $=\eta$ disperse $/ \eta$ continuous) [2].

$$
\mathrm{We}=0.25 \tau \times / \sigma
$$




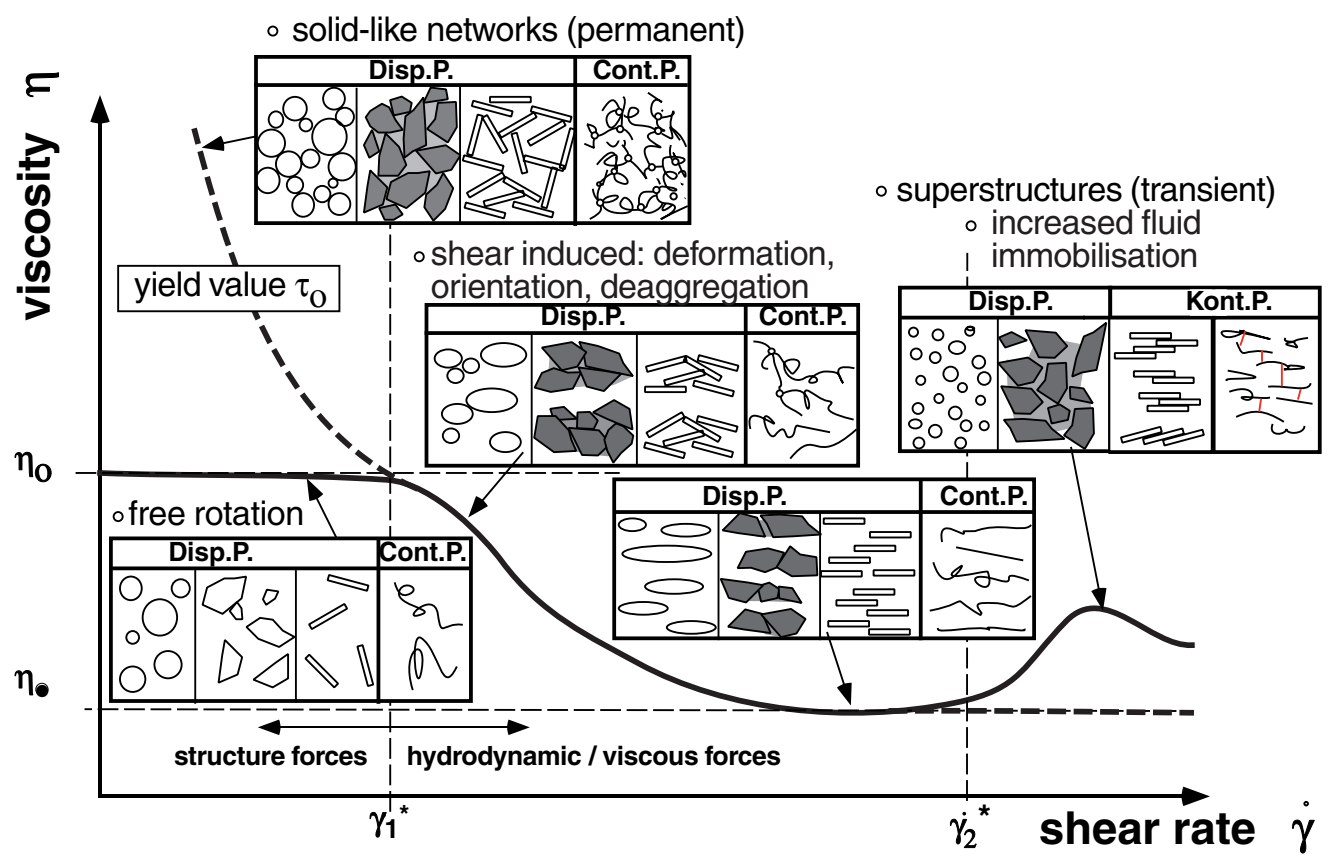

FIGURE 1: structure - rheology relationships for multiphase systems with disperse components (droplets, particles, fibres, macromolecules) [3]

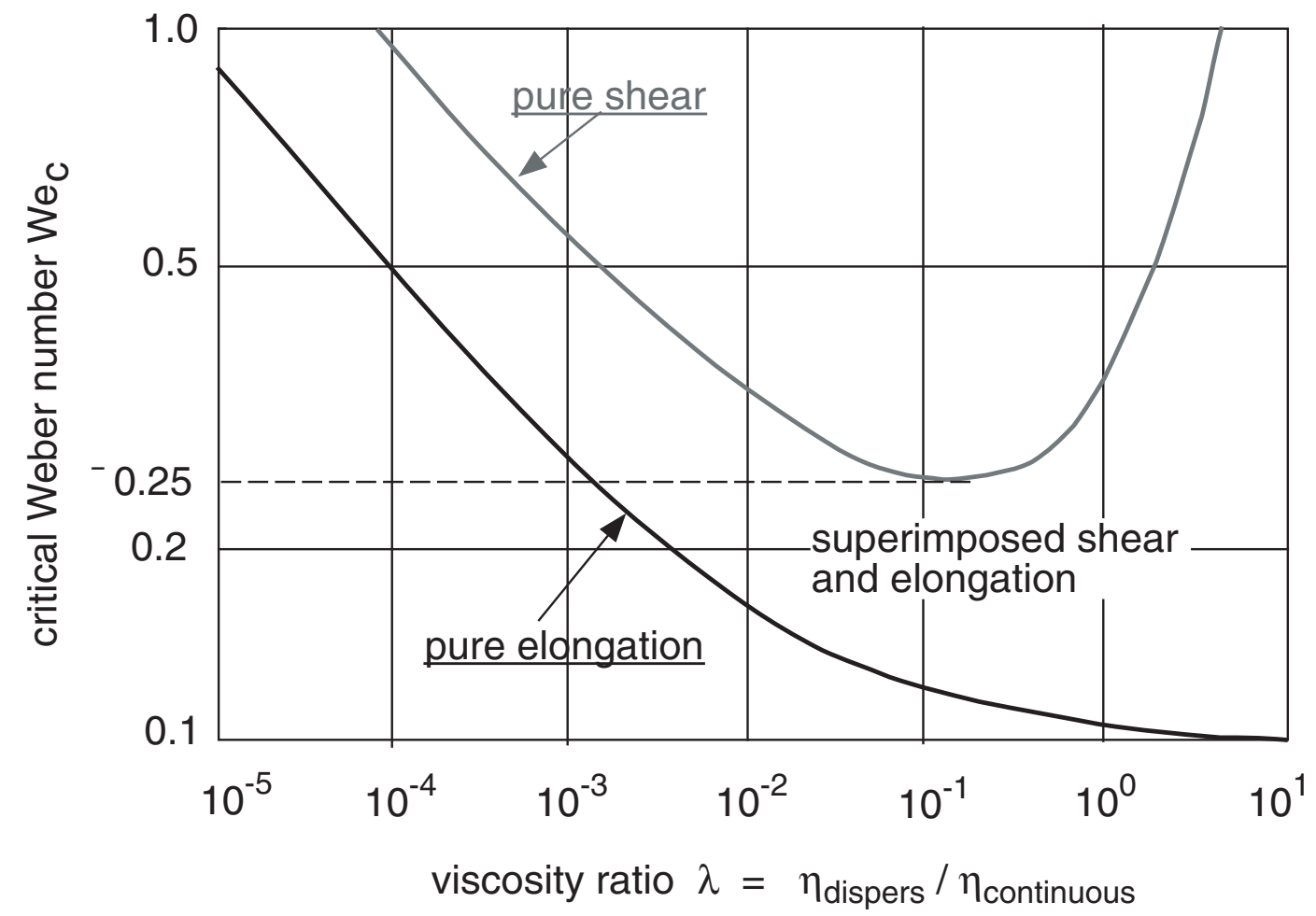

FIGURE 2: critical Weber number as a function of the viscosity ratio $\lambda=\eta_{\text {disperse }} / \eta_{\text {continuous }}$ for uniaxial shear- and elongational flows

Droplets are preferably used as "model structuring units" due to their isotropy, homogeneity and symmetry.In uni-axial shear the drop break-up is limited to the domain $\lambda \leq 4$, in uniaxial elongational flow fields, this limitation does not exist ( fig. 2) 


\section{EXPERIMENTS IN DISPERSING FLOW FIELDS}

\section{Model Flow Fields}

In order to get experimental results from laminar dispersing flow fields which are close to real flow fields in dispersing apparatus, the experimental set up should allow to investigate pure shear- and elongational flows but also their well defined superposition. These conditions are locally fulfilled in a Four-Roller Apparatus (FRA) and Cross-Slot Channels (CSC). These flow apparatus were constructed in such a way, that laser optics for optical rheometry, small angle light scattering (SALS) and microscopic / image analysis tools (e.g. CSLM) can easily be adapted. Within the FRA the velocity ratio $\alpha$ of the two diagonal roller pairs determines whether pure shear $(\alpha=0)$, pure elongational $(\alpha=1)$ or mixed flows of defined elongation to shear ratio $(0<\alpha \leq 0)$ are generated (see figure 3 ).
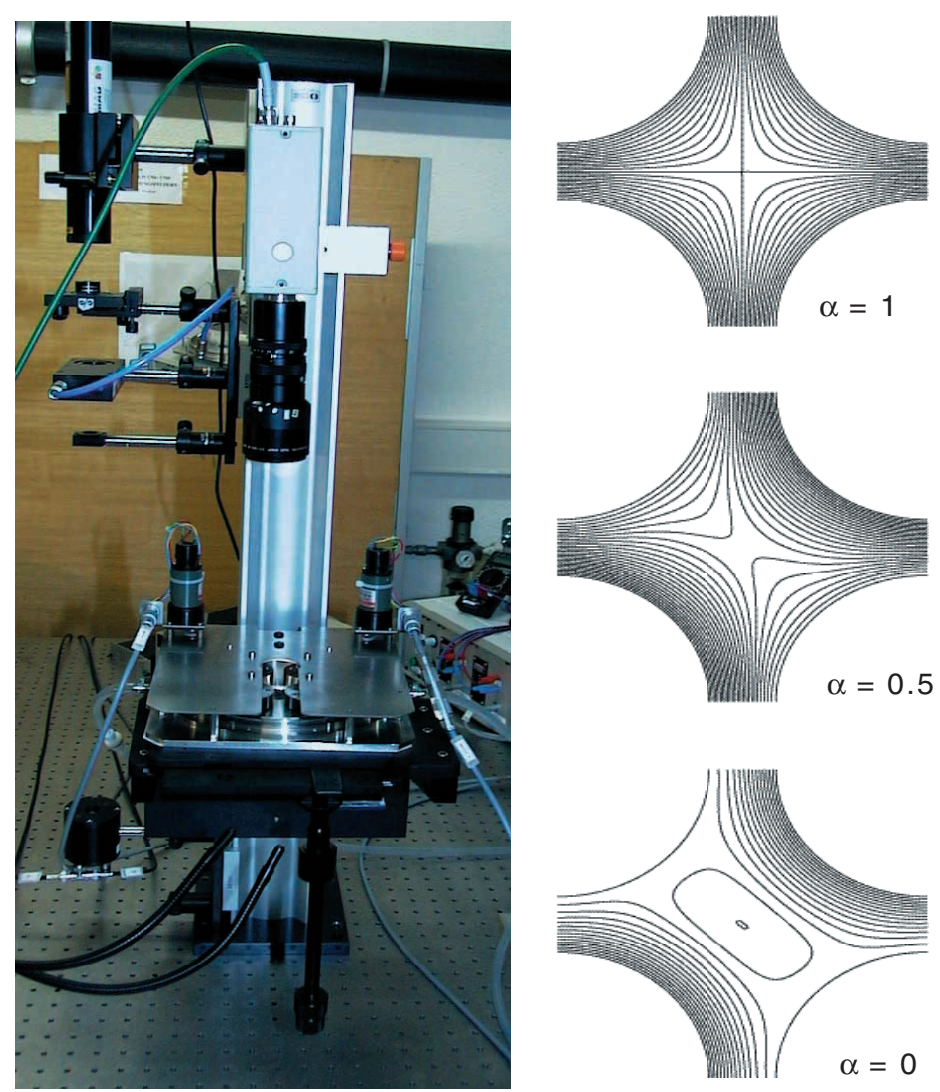

FIGURE 3: Four Roller Apparatus (FRA) connected to a laser optical set-up and stream line patterns for various diagonal roller pair speed ratios

Figure 3 shows the FRA built in a laser optic arrangement for optical rheometry. Figure 4 demonstrates drop deformation and break-up in in the stagnation flow domain of the FRA for two different viscosity ratios $\lambda$ under pure planar 
elongational flow conditions. Either drop break-up at the tips or the elongated drop filament break-up allow to generate very narrowly size distributed droplets.

\section{Single Drop Rheology}

From the dynamic drop behaviour during deformation and relaxation, interfacial elasticity and viscosity as well as interfacial tension can be estimated under small drop deformation conditions (interface ratio $\xi=\mathrm{A} / \mathrm{A}_{0} \leq 1.05$ ) as investigated in [4] and [5]. Equation 3 is derived for the drop relaxation from a mechanical spring/dashpot model of the drop interface [4].

$$
\begin{gathered}
\sigma=\frac{\dot{\gamma} x \eta_{c}}{2 D} \frac{19 \lambda+16}{16 \lambda+16} \\
A / A_{0}=\exp \left\{\frac{\tau^{2}\left(G_{G 1}+G_{G 2}\right)^{2}}{2 G_{G 1}{ }^{2} G_{G 2}{ }^{2}} \exp \left(-\frac{2 G_{G 1} G_{G 2}}{\left(G_{G 1}+G_{G 2}\right)\left(\eta_{d}+\eta_{c}\right)} t\right)\right\}
\end{gathered}
$$
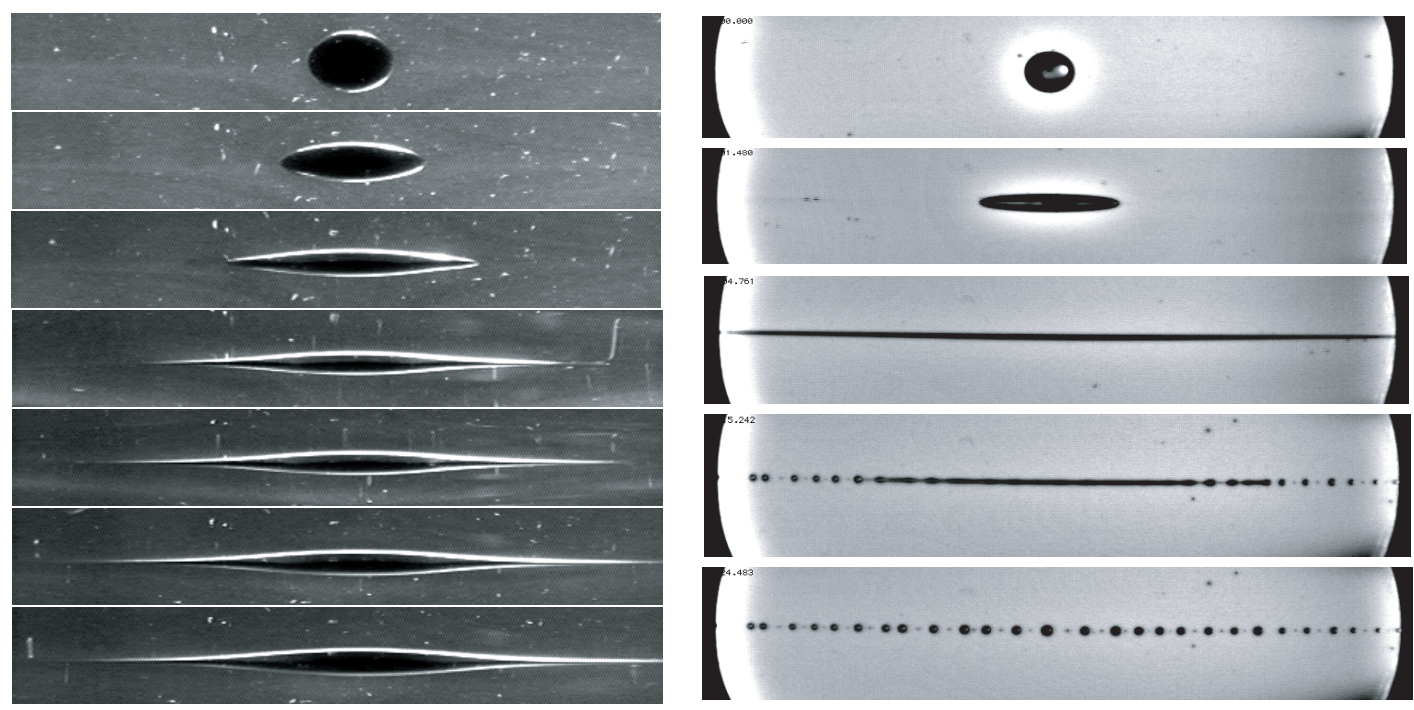

FIGURE 4: Droplet break-up under pure elongational flow conditions in a Four Roller Apparatus (FRA); left: tip-stream drop break-up for $\varepsilon \approx 5 \mathrm{~s}^{-1}, \lambda \approx 10^{-4}$ (water/silicon oil), right: filament breakup for $\varepsilon \approx 5 \mathrm{~s}^{-1}, \lambda \approx 1$ (water $+\mathrm{PEG} /$ silicon oil) 


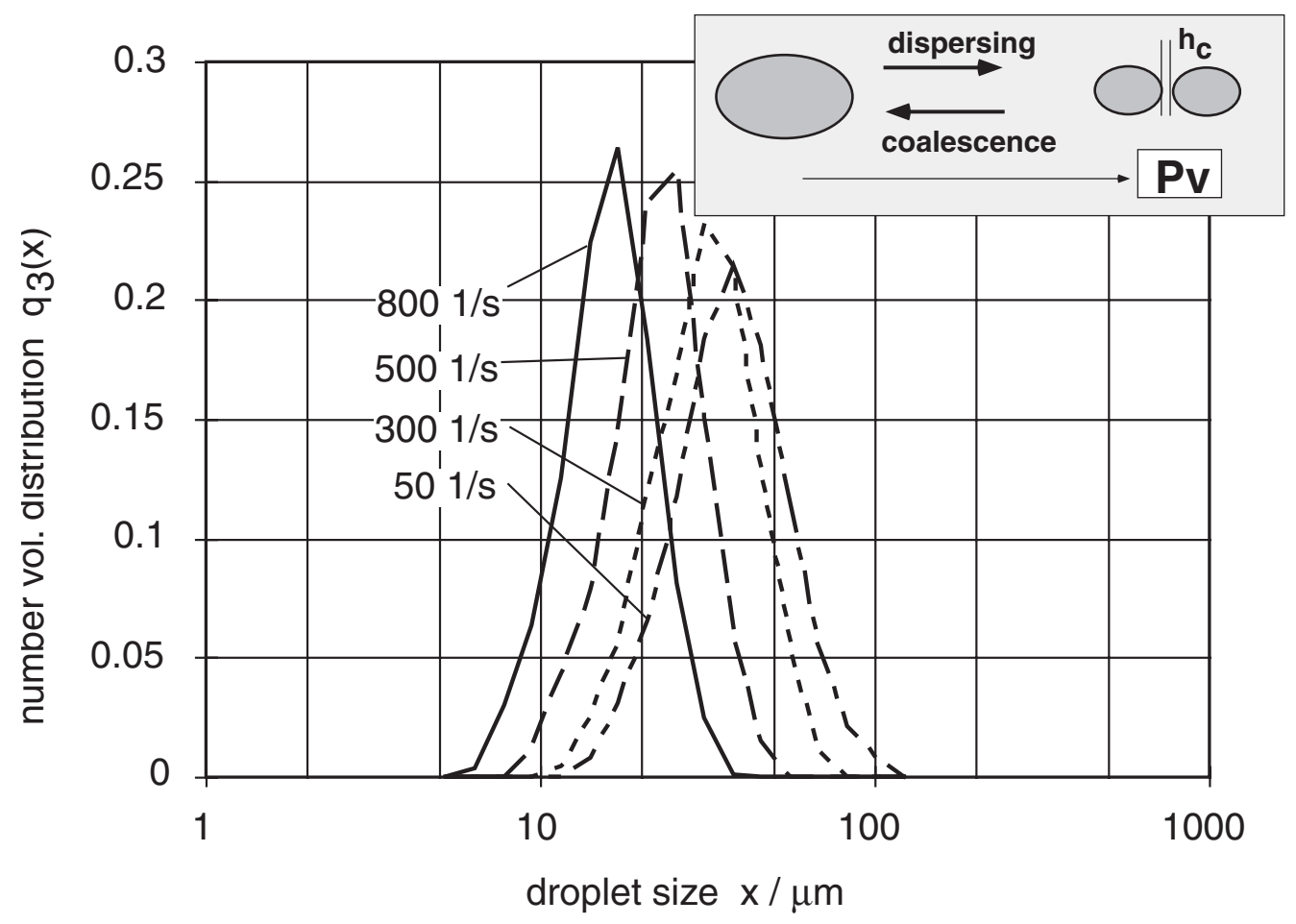

FIGURE 5: equilibrium drop size distributions after shear at various constant shear rates (pure uniaxial shear in concentric cylinder gap) [6]

\section{Emulsion Rheology}

Within multi-drop systems (emulsions) the disperse structure is determined by a dynamic equilibrium between drop break-up and -re-coalescence. Experimentally equilibrium drop size distributions were measured in pure shear flow fields (concentric cylinder gaps) as well as in superimposed shear and elongational flow fields within ex-centric cylinders for diluted $\left(\left(\phi_{\mathrm{V}} \leq 0.05\right)\right.$ and concentrated $\left(\phi_{\mathrm{V}}=\right.$ 0.3 ) model $\mathrm{O} / \mathrm{W}$ emulsions.

Figure 5 shows equilibrium drop size distributions from pure shear flow experiments. The related equilibrium viscosity function $\eta^{*}(\dot{\gamma})$ as well as so-called structure viscosity functions $\eta\left(\dot{\gamma}^{*}, \dot{\gamma}\right)$ (= viscosity functions at constant drop size, measured by step-shear experiments) are described elsewhere [7].

\section{MODELLING AND SIMULATIONS}

\section{Flow Simulation}

In order to get information about the locally acting shear- and normal stresses as well as the related shear- and elongational rates, Computational Fluid Dynamics (CFD) was applied to model flows in the geometries described before. Using a 


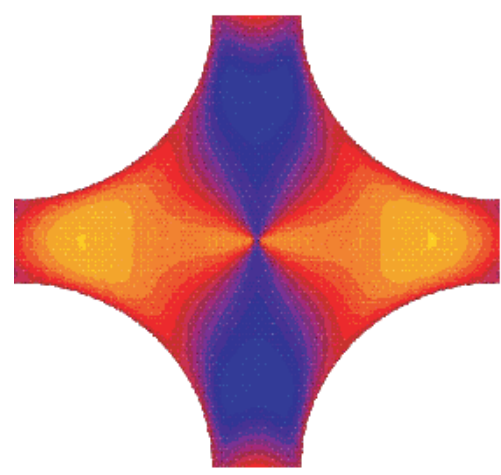

elongation rate

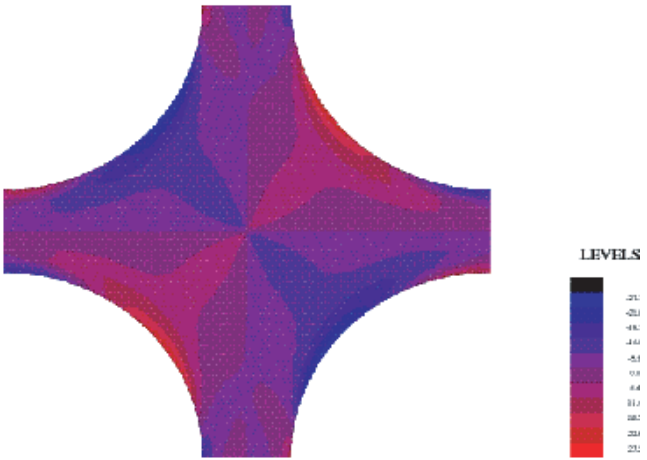

shear rate

FIGURE 6: CFD simulation of elongation (left) and shear rate fields (right) for a Four Roller Apparatus (FRA); (program SEPRAN) [8]

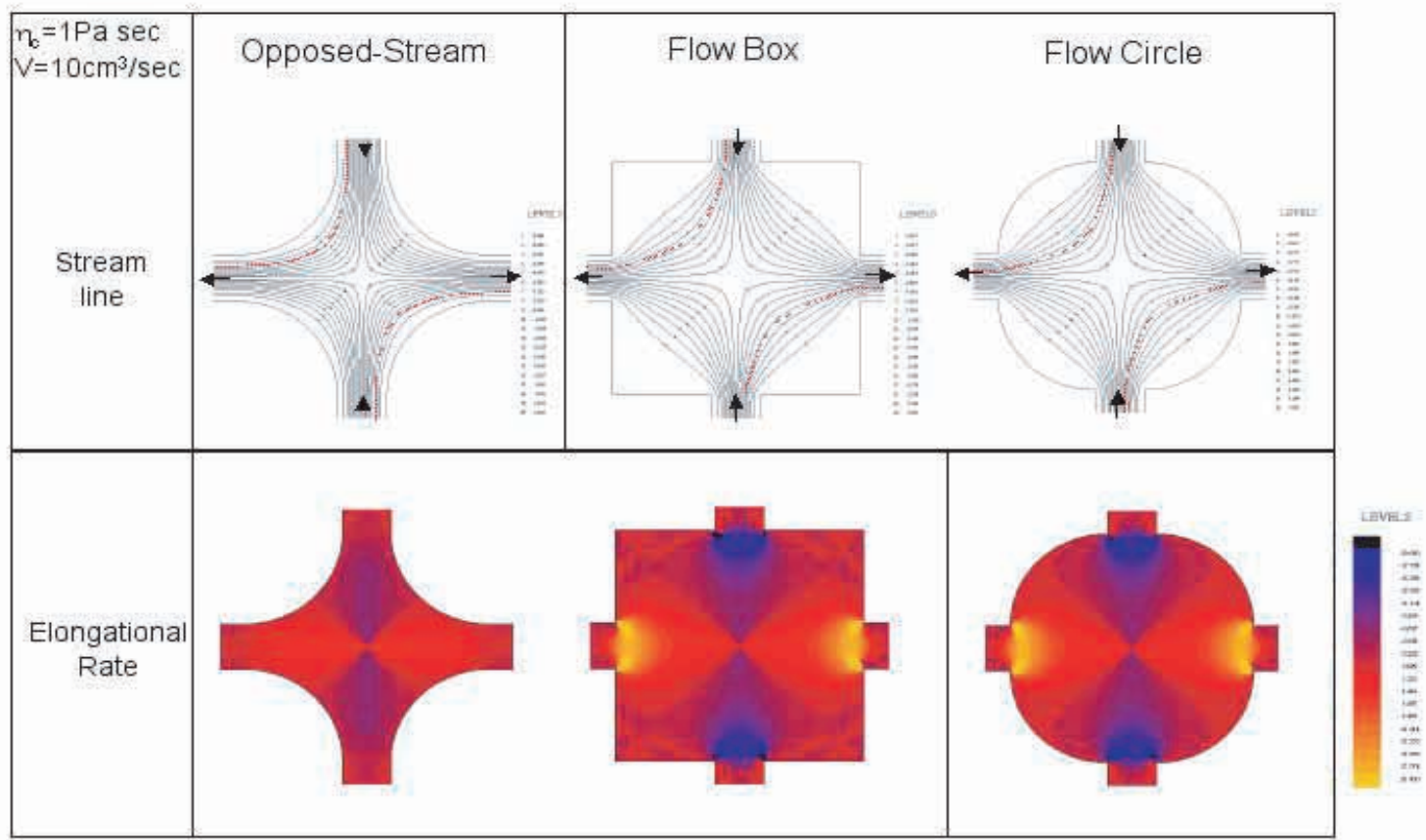

FIGURE 7: CFD simulation of streamlines (top) and elongation rate fields for different cross slot flow channels (program SEPRAN) 
partly modified finite element code (SEPRAN), the shear- and elongation rate fields were calculated by solving the typical equations for mass-, energy- and momentum conservation. Results are shown exemplary for the FRA in figure 6. Figure 7 demonstrates additionally elongation rate fields in three different experimentally investigated cross-slot flow channels. From such calculations the stress/strain history $(\mathrm{SSH})$ of droplets or other structuring elements respectively along flow tracks (particle tracks) within the apparatus flow, can be derived. The application of such a particle tracking calculation is demonstrated in the chapter where real rotor/stator-dispersing flow geometries are described.

\section{Simulation of Drop Deformation and Break-up}

Using a boundary integral element method (BIM) developed by Löwenberg et al.[9], the drop deformation in superimposed shear-/elongation flows was calculated, assuming clean interfaces (no surfactants). As the only interfacial property a constant equilibrium interfacial tension $\sigma$ was taken into account. Some results from such drop deformation simulations are demonstrated in figure 8 [10]. If critical capillary numbers $\mathrm{Ca}$. (=Weber numbers We) are derived from such calculations, the expected increase from pure elongation to pure shear is found as shown in figure 9.

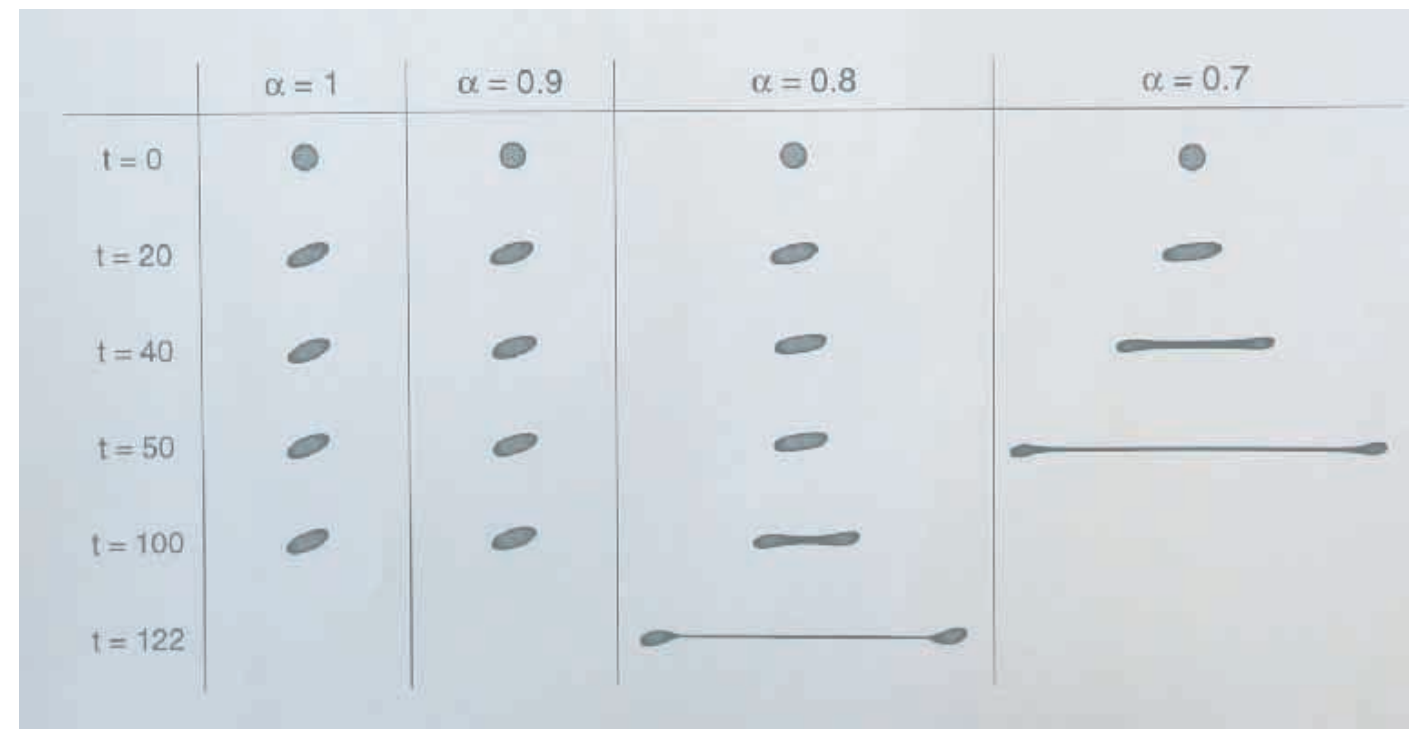

FIGURE 8: calculated droplet deformations under mixed shear-/elongational flow conditions $(\alpha=1$ : simple shear; $\alpha=0$ elongation); simulation with boundary $\mathrm{BIM}$; $\mathrm{We}=0.35, \lambda=1$ 


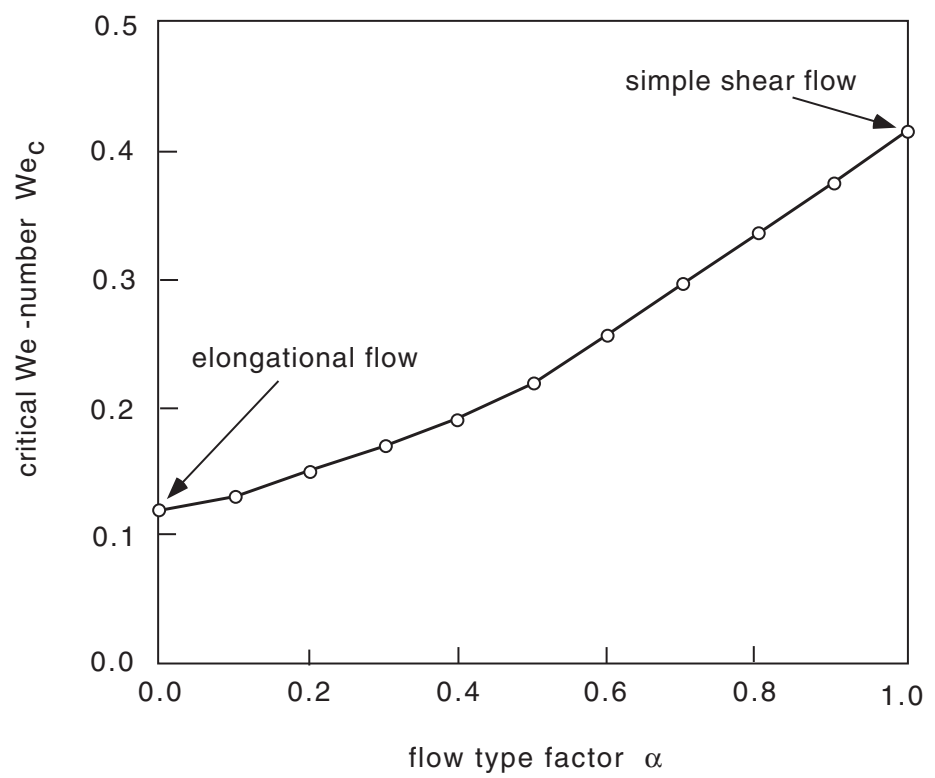

FIGURE 9: calculated critical Weber numbers (from BIM simulation); $\lambda=1$

\section{APPLICATIONS TO FOOD SYSTEMS}

As a consequence derived from the results of fundamental model dispersing flow investigations like described before, laminar dispersing flows to be applied in dispersing apparatus should be preferably of elongational nature. It is obvious that close to apparatus walls, at which the continuous phase adheres, shear always dominates elongation. However from practical experience even a minor elongational contribution is expected to have a relevant impact on the dispersing efficiency in particular at higher viscosity ratio $(\lambda>2)$ which shall quantified.

\section{Processing in continuous Narrow Annular Gap Reactors (NAGR)}

In narrow annular gap reactors (NAGR) which consist basically of a concentric cylinder gap geometry with rotating inner cylinder, the treated fluid systems experience a rather narrow range of shear stresses. Commonly dispersing/mixing elements are adapted to the rotating inner and /or the outer cylinder wall. In the case of in-built wall-scraping rotor- or stator blades or pins, which are in general inclined relative to a tangential plane to the cylinder wall (see figure 10), high elongation rates act locally. This has been proved experimentally using Particle Imaging Velocimetry (PIV) as well as by CFD calculations which applied a particle tracking method [11]. For the investigated flow gap between a rotating dispersing blade which is mounted to the inner cylinder scraping the surface of the outer cylinder, and the inner cylinder surface, three particle tracks are shown in figure 11. For various flow gap ratios of $\mathrm{r}_{\mathrm{S}}=0.25,0.4,0.6$ and a constant blade angle of $\beta=$ $30^{\circ}$, the calculated shear and elongation rates $\left(\dot{\gamma}, \dot{\varepsilon}_{1}\right)$ along the three selected particle tracks are shown in figure 12. 

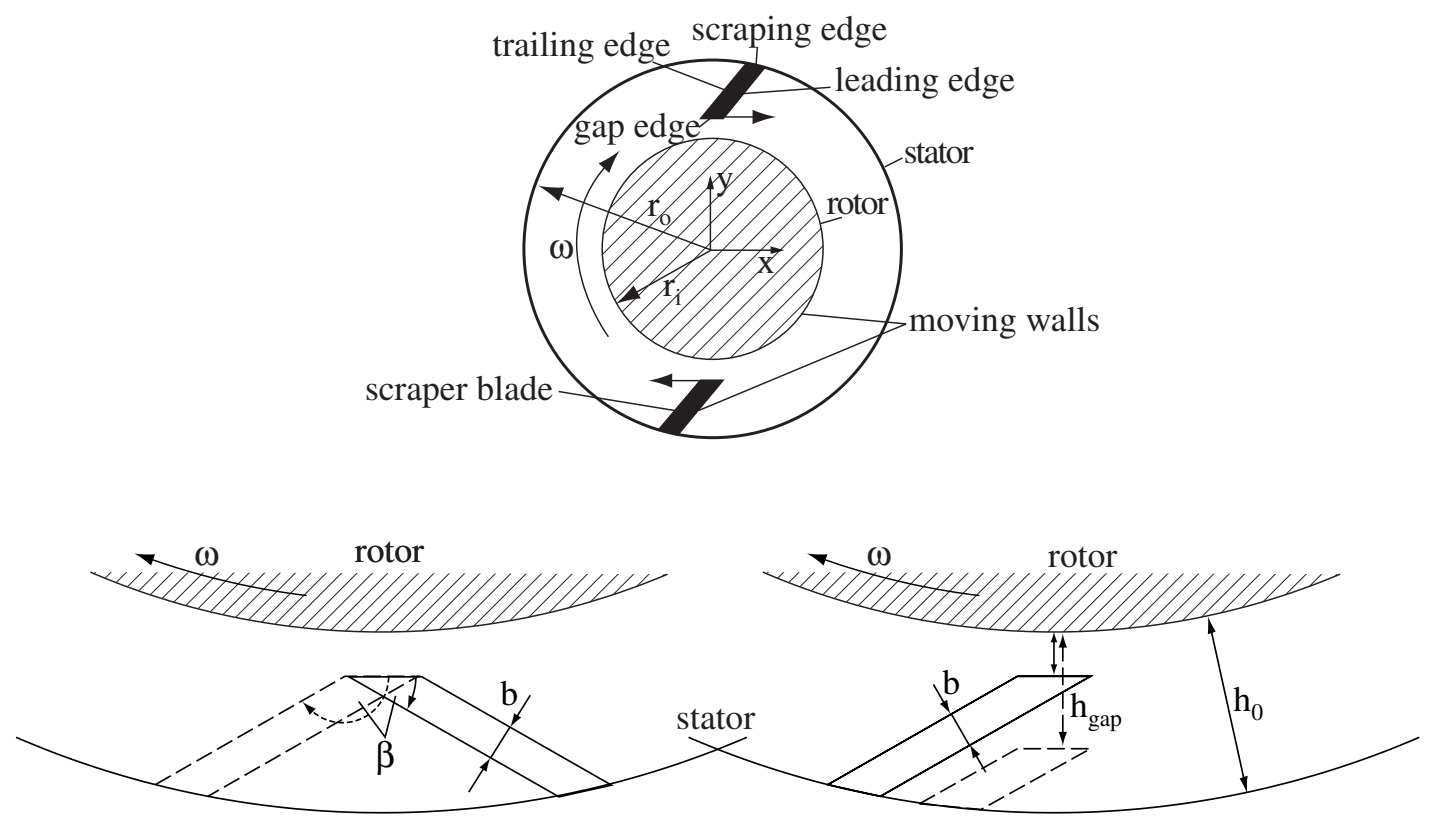

FIGURE 10: Cross section of narrow annular gap reactor (NAGR) with wall-scraping dispersing/mixing blade

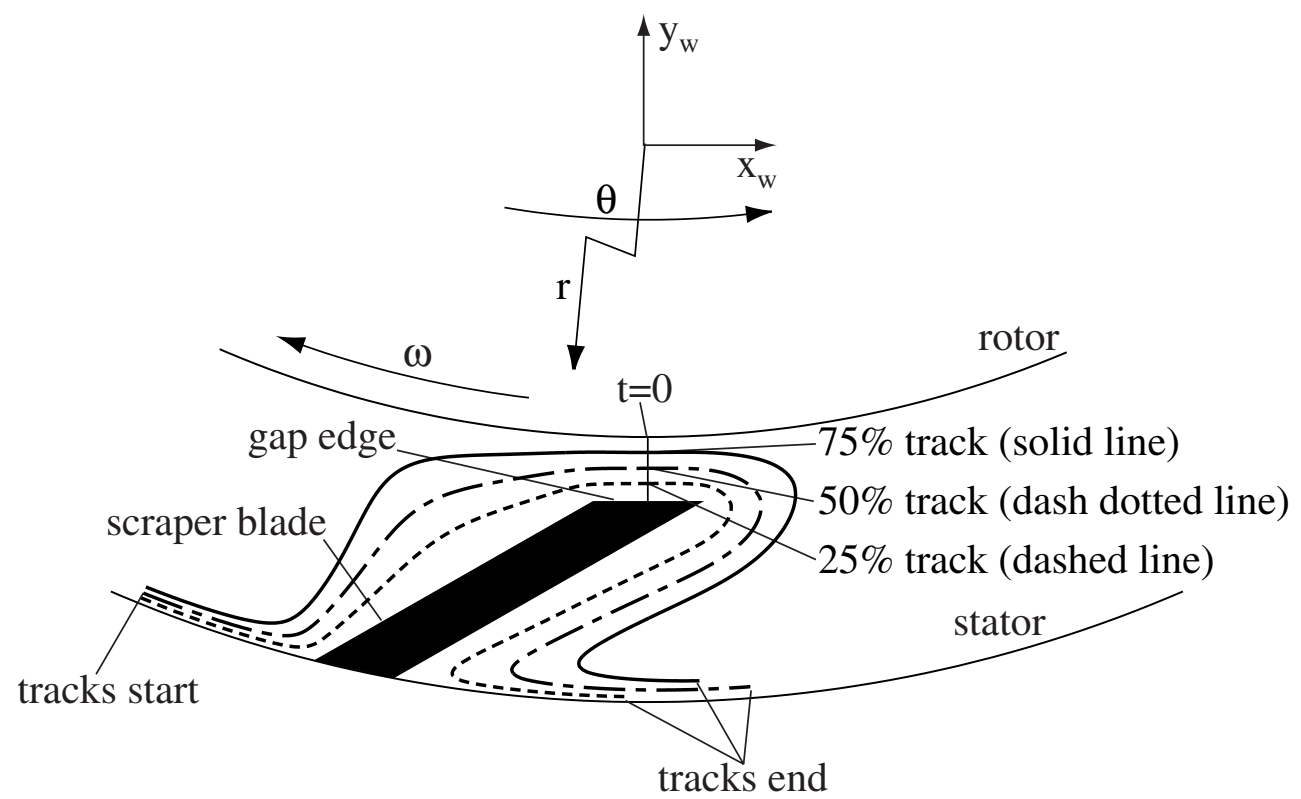

FIGURE 11: Selected particle tracks around dispersing/mixing blade in Narrow Annular Gap Reactor (NAGR) 

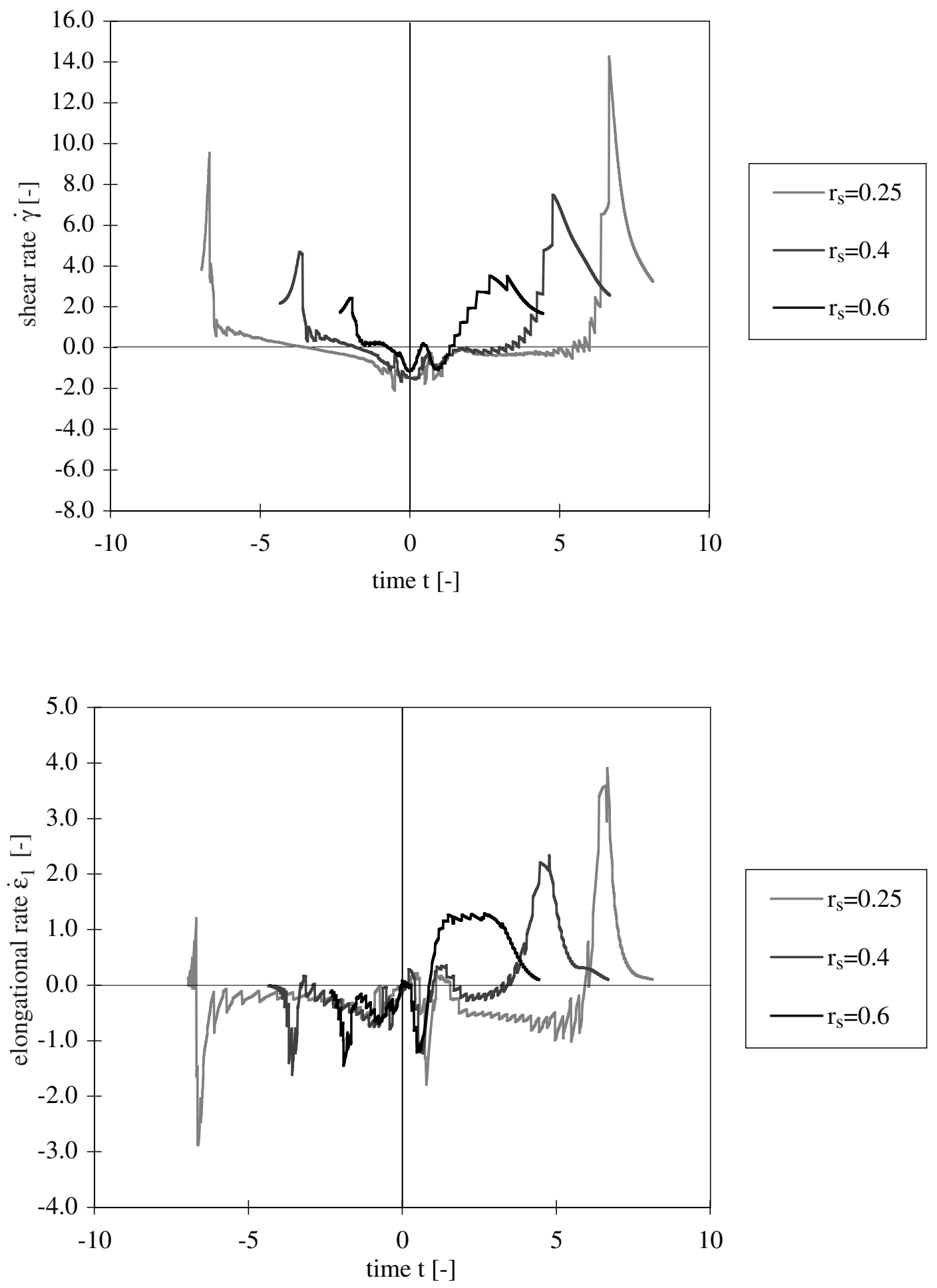

FIGURE 12 a,b: shear (top) and elongational rates (bottom) along selected particle tracks in Narrow Annular Gap Reactor (NAGR) with blade angle $\beta=30^{\circ} ; \mathrm{Re}=10$ and various scraper blade gap size ratio $\mathrm{r}_{\mathrm{S}}[11]$ 
FIGURE 13: Elongational deformation $\varepsilon_{1}$ (top) and shear deformation $\gamma$ (bottom) along selected particle tracks from $\mathrm{t}=\mathrm{t}_{\text {start }}$ to $\mathrm{t}=\mathrm{t}_{\text {end }} ; \operatorname{Re}=80, \mathrm{n}=0.65, \beta=150^{\circ}[12]$

Beside maximum shear- and elongation rates, the shearing and elongation times and consequently the total shear- and elongational deformations are considered in order to generate a flow induced (i.e. dispersed) microstructure.

Elongational and shear rate integrated over time provide the elongational and shear deformation $(\varepsilon, \gamma)$. Thus the calculated elongational rate $\dot{\varepsilon}_{1}$ and the shear rate $\dot{\gamma}$ with respect to the local $\left(\mathrm{e}_{1}, \mathrm{e}_{2}\right)$ coordinate system are integrated between $\mathrm{t}=\mathrm{t}_{\text {start }}$ and $\mathrm{t}=\mathrm{t}_{\mathrm{end}}$, as defined in Figure 10. Equations 4 and 5 describe the time integrals for $\varepsilon_{1}$ and $\gamma$, which are approximated numerically applying a trapezoidal rule according to Burden and Faires [13] using a time step $\Delta \mathrm{t}$, according to the time increments used for the numerical particle tracking (NPT).

$$
\begin{aligned}
& \varepsilon_{1}=\int_{t_{\text {start }}}^{t_{\text {end }}} \dot{\varepsilon}_{1}(t) d t \\
& \gamma=\int_{t_{\text {start }}}^{t_{\text {end }}} \dot{\gamma}(t) d t
\end{aligned}
$$

The total particle deformations $\varepsilon_{1}$ and $\gamma$ shown in figure 14 are compared for the three representative particle tracks, positioned at $25 \%, 50 \%$ and 75 for a shearthinning fluid $(n=0.65)$, with a flow incidences of $\beta=150^{\circ}$, at $\operatorname{Re}=80$.

For optimization of a structuring process the total energy density rates separately for the elongational and for the shear component are of further interest. The time integral of the elongational dissipation component in primary flow direction $e_{1}$ describes the elongational energy density $e_{E}$ (energy per volume), given with Equation 6, whereas the shear dissipation component contributes to the integral of the shear energy density $e_{S}$, as given with Equation 7 . The velocity vector is formulated in the polar coordinate system $(r, \theta)$. Applying the rotational coordinate transformation to the velocity gradient $\nabla_{\underline{v}}, \mathrm{e}_{\mathrm{E}}$ and $\mathrm{e}_{\mathrm{S}}$ are found in the local coordinate system $\left(\mathrm{e}_{1}, \mathrm{e}_{2}\right)$.

$$
\begin{gathered}
\mathrm{e}_{\mathrm{E}}=\int_{\mathrm{t}_{\text {start }}}^{\mathrm{t}_{\text {end }}} 2 \eta_{\mathrm{s}}(\dot{\gamma})\left[\left(\frac{\partial \mathrm{v}_{\mathrm{r}}}{\partial \mathrm{r}}\right)^{2}+\left(\frac{1}{\mathrm{r}} \frac{\partial \mathrm{v}_{\theta}}{\partial \theta}+\frac{\mathrm{v}_{\mathrm{r}}}{\mathrm{r}}\right)^{2}\right] \mathrm{dt} \\
\mathrm{e}_{\mathrm{S}}=\int_{\mathrm{t}_{\text {start }}}^{\mathrm{t}_{\text {end }}} \eta_{\mathrm{s}}(\dot{\gamma})\left[\frac{\partial \mathrm{v}_{\theta}}{\partial \mathrm{r}}+\frac{1}{\mathrm{r}} \frac{\partial \mathrm{v}_{\mathrm{r}}}{\partial \theta}-\frac{\mathrm{v}_{\theta}}{\mathrm{r}}\right]^{2} \mathrm{dt}
\end{gathered}
$$

Both Equations 5 and 6 are formulated in terms of the shear viscosity $\eta_{s}(\dot{\gamma})$ as a function of the shear rate $\dot{\gamma}$, which is transformed onto the local coordinate system 
$\left(\mathrm{e}_{1}, \mathrm{e}_{2}\right)(\dot{\gamma}$ is the magnitude of the rate-of-strain tensor $\underline{\underline{\gamma}}$, see Equation 3.36). For the investigated non Newtonian model fluid system with a watery $\mathrm{CMC}$ solution as the continuous fluid phase, $\eta_{\mathrm{S}}(\dot{\gamma})$ was described by a Carreau -Yasuda (CY) model defined by Equation 8.

$$
\frac{\eta-\eta_{\infty}}{\eta_{0}-\eta_{\infty}}=\left[1+\left(\lambda^{*} \dot{\gamma}\right)^{a}\right]^{(n-1) / a}
$$

Approximating the time integrals between $\mathrm{t}=\mathrm{t}_{\text {start }}$ and $\mathrm{t}=\mathrm{t}_{\mathrm{end}}$ applying a trapezoidal rule, the expressions $e_{E}$ and $e_{S}$ can be found numerically. The flow structuring shear- and elongation related volumetric energy inputs according to Equations 5 and 6 are also compared for the three representative particle tracks, positioned at $25 \%, 50 \%$ and $75 \%$ of the scraper blade gap width $\mathrm{h}_{\text {gap }}$. Figure 14 shows the increase of $e_{E}$ and $e_{S}$ as a function of time between $t=t_{\text {start }}$ and $t=t_{\text {end }}$ along the three representative particle tracks for a shear-thinning fluid $(n=0.65)$ with $\beta=150^{\circ}$ at $\operatorname{Re}=80$. All elongational and shear energies commonly develop in a step function manner. This behaviour is caused by the pronounced turning sections of the tracks around the scraper blade.

Summarizing the CFD results it can be stated that the main influence on the deformation energy depicts the flow incidence, which varies the total average elongational energy by about $95 \%$ between blade angles of $\beta=30^{\circ}$ and $\beta=110^{\circ}$. The maximum change for the total average shear energy at $\beta=90^{\circ}$ and $\beta=110^{\circ}$ is calculated as $33 \%$.

Comparing the impact of the rotor velocity with the influence of the flow incidence, variations of the scraper blade angle cause higher structuring anisotropy with respect to elongational and shear strain, than increasing the Reynolds number between 10 and 80. All flow cases show larger total shear energies than total elongational energies. The minimum difference is found for $\operatorname{Re}=10, \beta=30^{\circ}, \mathrm{n}=0.65$ with a factor of 3.5

In order to express the flow structuring energy contributions of the two distinguished flow types (shear, elongation), Equations 9 and 10 define the (local) flow type contribution factors $\chi_{\mathrm{E}}, \chi_{\mathrm{S}}$ with regard to the investigated scraper blades, for elongational flow $\chi_{\mathrm{E}}$ and shear flow $\chi_{\mathrm{S}}$, respectively.

$$
\begin{aligned}
& \chi_{\mathrm{E}}=\frac{\mathrm{e}_{\mathrm{E}}}{\mathrm{e}_{\mathrm{tot}}} \\
& \chi_{\mathrm{S}}=\frac{\mathrm{e}_{\mathrm{S}}}{\mathrm{e}_{\mathrm{tot}}}
\end{aligned}
$$

$\chi_{\mathrm{E}} \geq 0.14$ which was the lowest contribution factor for the elongational flow type showed already a qualitative improvement of the dispersing efficiency (i.e. drop size reduction) at constant energy input, compared to a pure shear flow field (concentric cylinder gap). To quantify the improvement of dispersing efficiency as a 
function of $\chi_{\mathrm{E}}$ experimentally in more detail, is subject of our ongoing research work in this field. Within the work reported here the maximum values of $\chi_{\mathrm{E}}=0.35$ was received for $\operatorname{Re}=10, \beta=150^{\circ}, \mathrm{r}_{\mathrm{S}}=0.6 ; \mathrm{n}=0.65$.
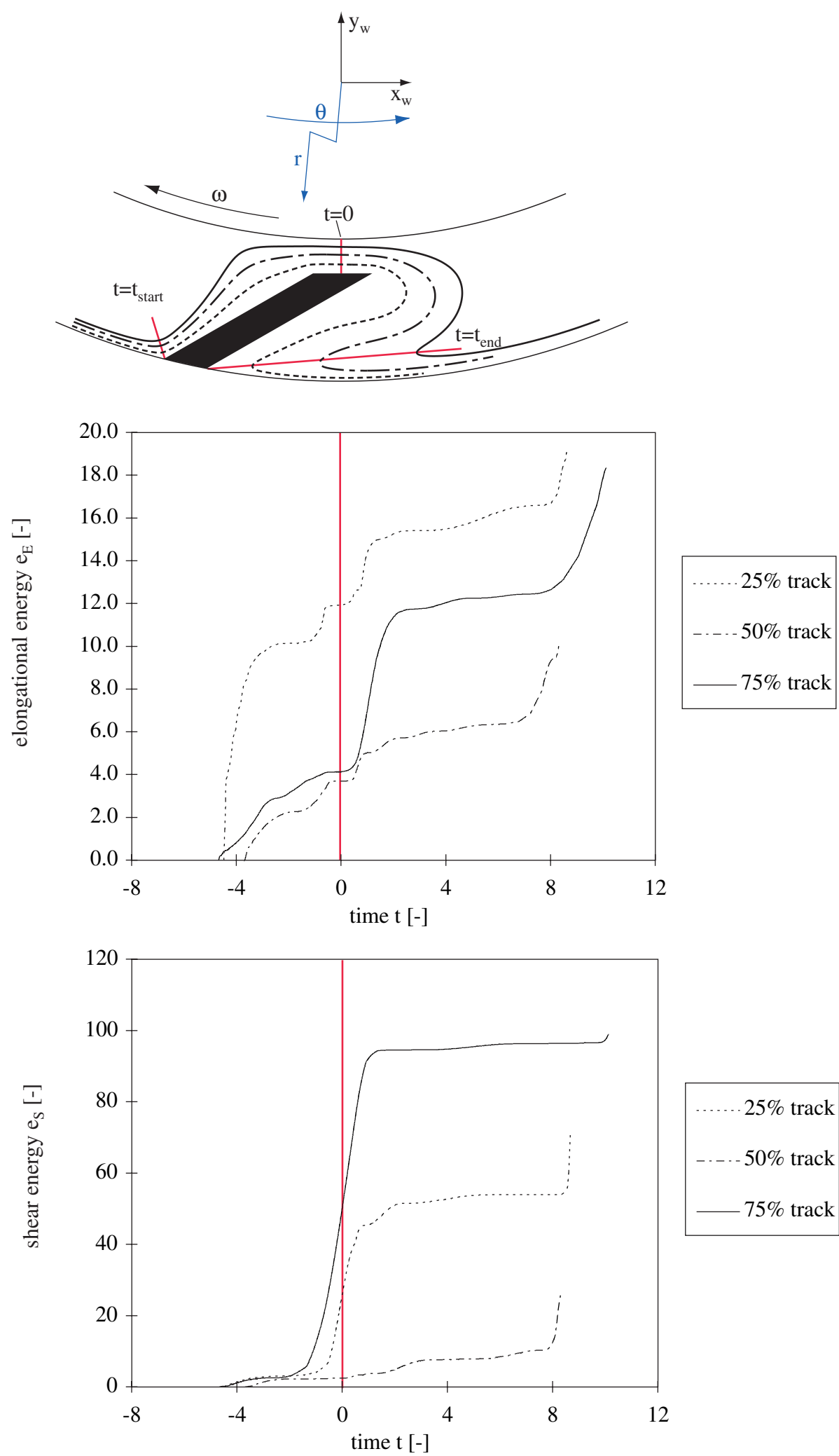

..... 25\% track
$-.50 \%$ track
$-75 \%$ track 
FIGURE 14: Volumetric elongational energy $\mathrm{e}_{\mathrm{E}}$ (top) and shear energy $\mathrm{e}_{\mathrm{S}}$ (bottom) for selected particle tracks from $\mathrm{t}=\mathrm{t}_{\text {start }}$ to $\mathrm{t}=\mathrm{t}_{\text {end }} ; \mathrm{Re}=80, \mathrm{n}=0.65 ; \beta=150^{\circ}[12]$

\section{Development of multiphase food products with shaped particles}

As demonstrated in model flow experiments described in the previous chapters, the application of well defined shear and in particular elongational flow fields allows to shape disperse components like immiscible fluid droplets. If such disperse fluid components reach a certain shape during the flow structuring procedure (i.e. a certain equilibrium shape by balancing flow stresses and Laplace pressure, which is proportional to the interfacial tension), shape fixing was applied. This has been done by changing temperature or adding skin building agents $[14,15]$.

For different two phase fluid/biopolymer systems (i.e. gelatine (w) /oil; gelan/אcarraghenan) drop shaping experiments were carried out within pure shear (concentric cylinder gap) and mixed shear/elongation flow fields (FRA, excentric cylinder gap). Resulting shape fixed particle structures are shown in figure 15. If the shaped particles are separated by centrifugation and then mixed with a fluid at constant volume fraction a shape-related rheological behaviour is detected.

If ellipsoidally or fibre-like shaped particles with a large aspect ratio $(\mathrm{L} / \mathrm{D} \geq 10)$ are mixed into a continuous fluid with a solids fraction of about $\phi_{\mathrm{V}} \geq 0.15$, a strong network results for the randomly oriented particles. Rheologically a yield value is exhibited.

If such systems are sheared and/or elongated particle orientation in the shear/elongation flow direction is induced. This reduces the viscosity strongly. In the concentrated system a further particle rotation is hindered by the oriented neighbour particles.
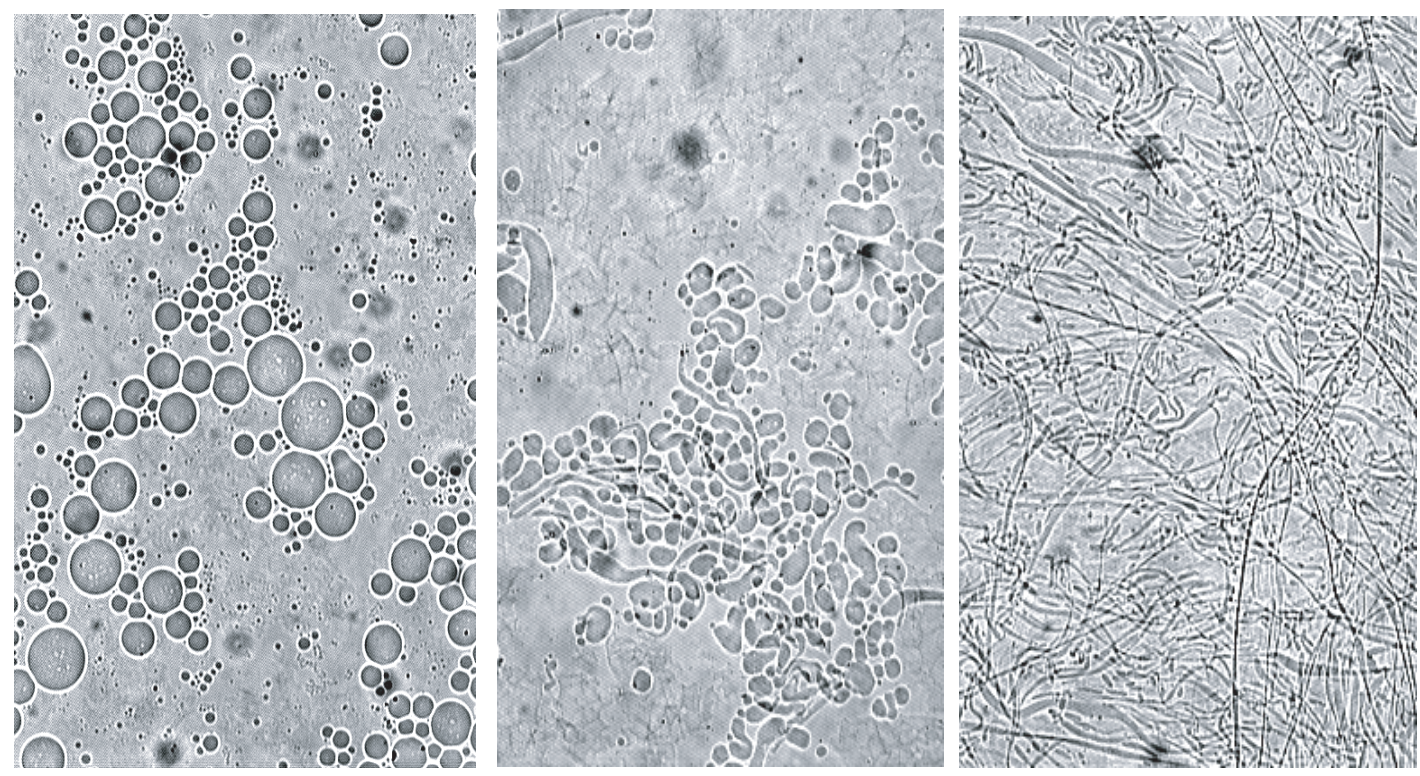
FIGURE 15: Shape-adjusted biopolymer particles (gellan) in another continuous watery biopolymer solution ( $\kappa$-carraghenan); shear / elongation induced structuring in excentric cylinder gap at increasing shear and elongation rates (from left to right)

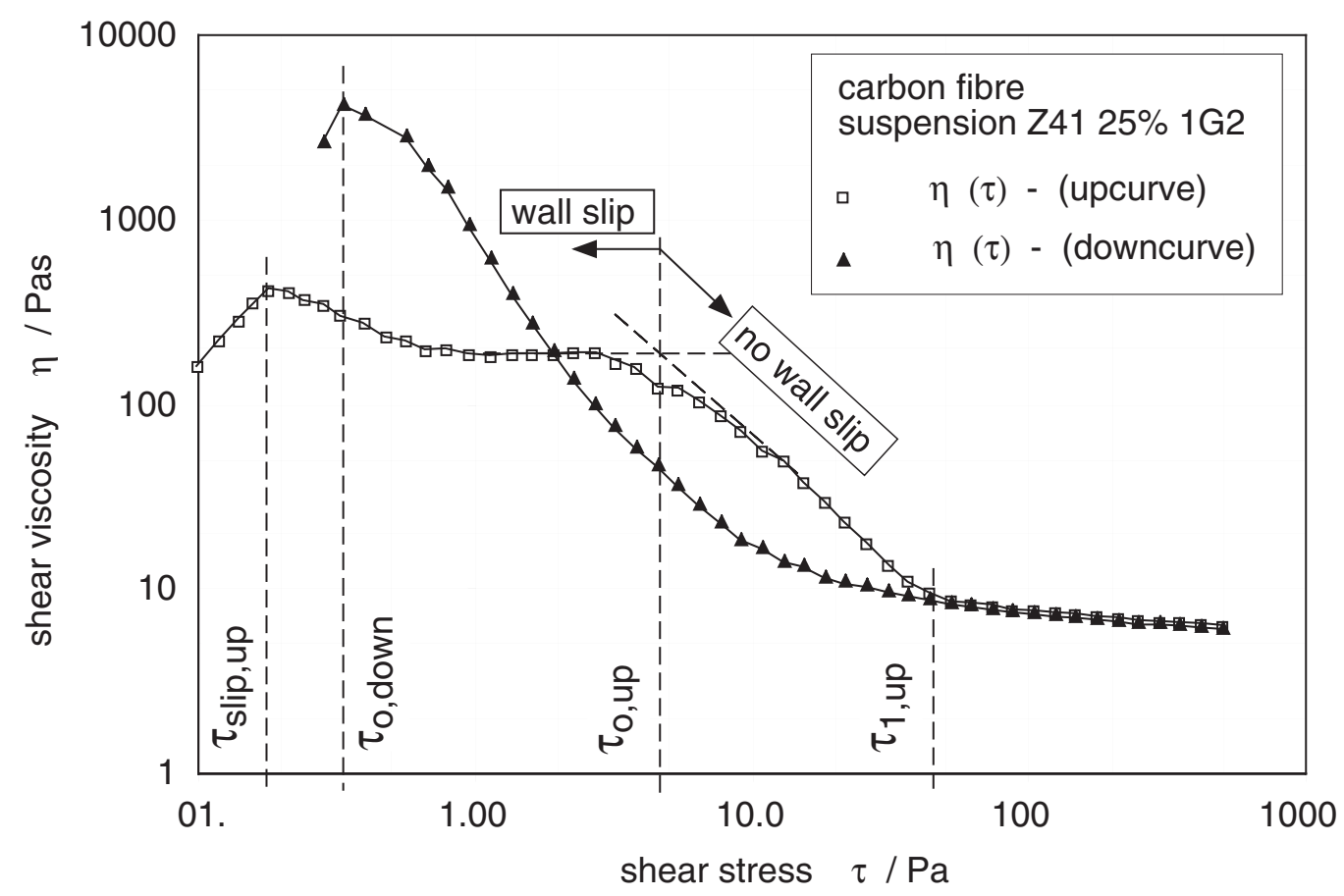

FIGURE 16: Shear viscosity functions (up-/down curves) for a concentrated fibre suspension system; influence of fibre orientation [15]

For a model suspension with fibre-like particles figure 16 demonstrates the impact of particle orientation on the viscosity function investigated by up-/downcurve shear flow measurements. If the particles are small enough $(<1 \mu \mathrm{m})$ Brownian motion related diffusion can restructure the fluid system randomly. If this principle is applied to food products gel-type systems can be created which i.e. get liquid if they are sheared between tongue and plate or if they are stirred, and which solidify reversibly by heating.

\section{List of Symbols}

$\begin{array}{llll}\eta_{\mathrm{o}} & \text { zero shear viscosity } & \lambda^{*} & \text { time constant } \\ \eta_{\ldots} & \text { Upper qu.Newt. viscosity } & \mathrm{n} & \text { power law index } \\ \mathrm{G}_{\mathrm{G} 1} & \text { interfacial tension modulus } & \mathrm{e} 1 & \text { elongation deformation } \\ \mathrm{G}_{\mathrm{G} 2} & \text { interfacial skin elasticity } & \alpha & \text { shear/elong. flow ratio } \\ \tau_{0} & \text { yield value } & \eta^{*}(\dot{\gamma}) & \text { equil. viscosity function }\end{array}$




$\begin{array}{llll}\tau & \text { shear stress } & \eta\left(\dot{\gamma}^{*}, \dot{\gamma}\right) & \text { Structure viscosity fct. } \\ \sigma & \text { interfacial tension } & \dot{\gamma}^{*} & \text { shear structure index } \\ \mathrm{x} & \text { particle diameter } & \mathrm{A}_{0} & \text { spherical droplet surface } \\ \lambda & \text { viscosity ratio (droplet) } & \mathrm{A} & \text { deformed droplet surface } \\ \eta_{\mathrm{d}} & \text { disperse phase viscosity } & \gamma & \text { Shear deformation } \\ \eta_{\mathrm{c}} & \text { continuous phase viscosity } & \dot{\gamma} & \text { shear rate }\end{array}$

\section{REFERENCES}

1. Windhab E. 2000."Fluid Immobilization-A structure related key mechanism for the viscous flow behaviour of concentrated suspension systems ", in P.Fischer, I.Marti, E.Windhab: Proc. of $2^{\text {nd }}$ Int. Symp. Of Food Rhreology and Structure; 12-16.3.2000, Zürich; pp. 13-24

2. Grace, H.P. 1982. "Dispersion Phenomena in High Viscosity Immiscible FluidSystems", Eng. Found. Res. Conference Mixing, $3^{\text {rd }}$ Andover, N.H.; Republished 1982 in Chem. Eng.Commun. 14, 225-277

3. Windhab E.1996. "The influence of structure/rheology relationsships on the processing of food suspensions Annual Transactions of the Nordic Rheological Society, Vol.4; 3-15; 1996

4. Wolf B.; 1995.”Untersuchungen zum Formverhalten mikroskopisch kleiner Fluidtropfen in stationären und instationären Scherströmungen”, PhD Thesis, ETH Zürich, 1995, Nr. 11068

5. Cox R.G.;1969. Journal of Fluid Mechanics, 37, pp. 601-623

6. Windhab, E.; Wolf, B.1992.'Influence of Deformation and Break-up for Emulsified Droplets on the Rheological Emulsion Properties", Theoretical and Applied Rheology, edited by P. Moldenaers and R. Keunings Proc. XIth Int. Congr. on Rheology, Brussels, Belgium, August 17-21, vol. 2, Seite 681-683, 1992

7. Windhab E. 1988."A new method for describing the time dependent rheological behaviour of concentrated suspension", Suppl. To Rheologica Acta; Progress and trends in Rheology II, Steinkopf Verlag Darmstadt, 1988

8. Kaufmann S., Fischer P., Windhab E.2000.'Investigation of Droplet Dispersing Processes in Shear and Elongational Flow", in P.Fischer, I.Marti, E.Windhab: Proc. of $2^{\text {nd }}$ Int. Symp. Of Food Rhreology and Structure; 12-16.3.2000, Zürich; pp. 404-406

9. Christini V., Blawzdziewicz J., Löwenberg M.1998.”Drop break-up in three-dimensional viscous flow", Physics of Fluids 10(8),1998; pp. 1781-1783

10. Feigl K. 2000."Use of Numerical Simulation in Food Processing”, in P.Fischer, I.Marti, E.Windhab: Proc. of $2^{\text {nd }}$ Int. Symp. Of Food Rhreology and Structure; 12-16.3.2000, Zürich; pp. 69-76

11. Stranzinger M.1999."Numerical and Experimental Investigations of Newtonian and NonNewtonian Flow in Annular Gaps with Scraper Blades", PhD Thesis, ETH Zürich (1999); Nr. 13369 
12. Bieder A., Stranzinger M., Windhab E.2000.’Einfluss der Prozessgeometrie auf das Strukturierungsverhalten in Ringspaltreaktoren”, Semesterwor ETH Zürich 2000

13. Burden R.L., Faires J.D. 1993.”Numerical Analysis, $5^{\text {th }}$ Edition edn. PWS Publishing Company

14. R. Scirocco, B. Wolf, P. Fischer, E. Windhab.2000.”, Diploma Thesis, ETH-Zürich

15. Wolf B. 2000.'Biopolymer Suspensions with Spheroidal and Cylindrical Particle Shapes: Generation and Flow Behaviour", in P.Fischer, I.Marti, E.Windhab: Proc. of $2^{\text {nd }}$ Int. Symp. Of Food Rhreology and Structure; 12-16.3.2000, Zürich; pp. 404-406

16. Eischen J.C. 1999.'Bildanalytische und rheologische Untersuchungen zum Orientierungs- und Strukturierungsverhalten von faserförmigen Partikeln in laminaren Scherströmungen”, $\mathrm{PhD}$ Thesis, ETH-Zürich,1999; Nr. 13217 